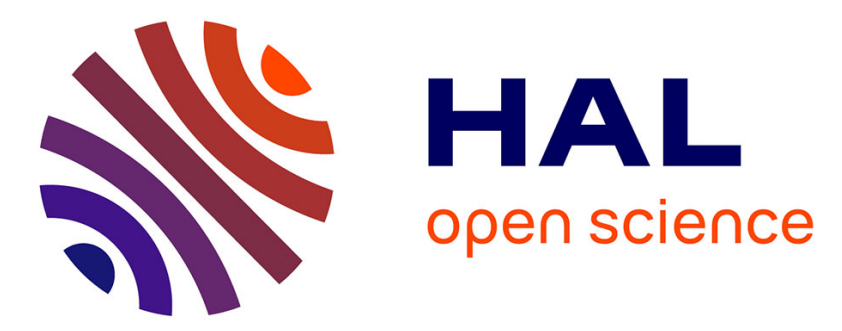

\title{
$\mu$-diff: A matlab toolbox for multiple scattering problems by disks
}

Bertrand Thierry, Xavier Antoine, Chokri Chniti, Hasan Alzubaidi

\section{To cite this version:}

Bertrand Thierry, Xavier Antoine, Chokri Chniti, Hasan Alzubaidi. $\mu$-diff: A matlab toolbox for multiple scattering problems by disks. ICNAAM 2014, Sep 2014, Rhodes, Greece. pp.390002, 10.1063/1.4912612 . hal-01273771

\section{HAL Id: hal-01273771 \\ https://hal.science/hal-01273771}

Submitted on 13 Feb 2016

HAL is a multi-disciplinary open access archive for the deposit and dissemination of scientific research documents, whether they are published or not. The documents may come from teaching and research institutions in France or abroad, or from public or private research centers.
L'archive ouverte pluridisciplinaire HAL, est destinée au dépôt et à la diffusion de documents scientifiques de niveau recherche, publiés ou non, émanant des établissements d'enseignement et de recherche français ou étrangers, des laboratoires publics ou privés. 


\title{
$\mu$-diff: a Matlab toolbox for multiple scattering problems by disks
}

\author{
Bertrand Thierry*, Xavier Antoine ${ }^{\dagger}$, Chokri Chniti** and Hasan Alzubaidi** \\ ${ }^{*}$ Laboratoire J.L. Lions (LJLL), University of Paris VI, Paris, France. \\ †Université de Lorraine, IECL, UMR 7502, Vandoeuvre-lès-Nancy, F-54506, France. \\ **Department of Mathematics, University College in Qunfudah, Umm Al-Qura University, Saudi Arabia.
}

\begin{abstract}
The aim of this paper is to describe a Matlab toolbox, called $\mu$-diff, which is designed for spectrally solving twodimensional complex multiple scattering by a large collection of circular cylinders. The formulation is based on the Fourier series expansions of the four basic integral operators arising in scattering problems. Based on these expressions, a spectrally accurate finite-dimensional solution of multiple scattering problems can be simply obtained for complex media even when many scatterers are considered as well as large frequencies. The efficient solution of the final linear system to solve makes use of preconditioned Krylov subspace solvers for block Toeplitz matrices.
\end{abstract}

Keywords: multiple scattering, disks, integral equations

PACS: $31 \mathrm{~A} 10,74 \mathrm{~J} 20,65 \mathrm{~N} 35$

\section{INTRODUCTION}

Let us consider $M$ regular, bounded and disjoint scatterers $\Omega_{p}^{-}, p=1, \ldots, M$, distributed in $R^{2}$, with boundary $\Gamma_{p}:=\Omega_{p}^{-}$. The scatterer $\Omega^{-}$is defined as the collection of the $M$ separated obstacles, i.e. $\Omega^{-}=\cup_{p=1}^{M} \Omega_{p}^{-}$, with boundary $\Gamma=\cup_{p=1}^{M} \Gamma_{p}$. The homogeneous and isotropic exterior domain of propagation is $\Omega^{+}=R^{2} \backslash \overline{\Omega^{-}}$. For the sake of conciseness, we assume in the presentation that the scatterers are sound-soft (Dirichlet boundary condition), but other situations can be handled by $\mu$-diff (e.g. sound-hard scatterers, impedance boundary conditions, penetrable scatterers). We now consider a time-harmonic incident plane wave $u^{i n c}(\mathbf{x})=e^{i k \Theta \cdot \mathbf{x}}$ (with $\left.\mathbf{x}=\left(x_{1}, x_{2}\right) \in R^{2}\right)$ illuminating $\Omega^{-}$, with an incidence direction $\Theta=(\cos (\theta), \sin (\theta))$ and a time dependence $e^{-i \omega t}$, where $\omega$ is the wave pulsation and $k$ is the wavenumber. The multiple scattering problem of $u^{i n c}$ by $\Omega^{-}$consists in computing the scattered wavefield $u$ as the solution to the boundary-value problem [1]

$$
\begin{aligned}
& \left(\Delta+k^{2}\right) u=0, \text { in } \Omega^{+}, \\
& u=-u^{i n c}, \text { on } \Gamma, \\
& \lim _{|\mathbf{x}| \rightarrow+\infty}|\mathbf{x}|^{1 / 2}\left(\nabla u \cdot \frac{\mathbf{x}}{|\mathbf{x}|}-i k u\right)=0 .
\end{aligned}
$$

The operator $\Delta=\partial_{x_{1}}^{2}+\partial_{x_{2}}^{2}$ is the Laplace operator and $\left(\Delta+k^{2}\right)$ is the Helmholtz operator. The gradient operator is $\nabla$ and $|\mathbf{x}|=\sqrt{\mathbf{x} \cdot \mathbf{x}}$, where $\mathbf{x} \cdot \mathbf{y}$ is the scalar product of two vectors $\mathbf{x}$ and $\mathbf{y}$ of $R^{2}$. The last equation of (1) is the well-known Sommerfeld's radiation condition at infinity that ensures the uniqueness of $u$.

The toolbox $\mu$-diff uses the powerful integral equation formulation methods for solving scattering problems. Being able to use integral operators allows us to formulate the solution to a given scattering problem by using traces theorems and variational approaches. When the boundary $\Gamma$ is general, then boundary element discretization techniques are required. Even if these methods are very useful for general shapes, they also have some disadvantages. First, they lead to large full linear systems to solve, most particularly when investigating small wavelength problems $(\lambda \ll 1)$ and large scatterers or collections of many scatterers $(M \gg 1)$. These systems need a lot of memory storage and their solution is highly time consuming. The solution can be accelerated by using Krylov subspace solvers [2] in conjunction with fast matrix-vector products algorithms (for example Multilevel Fast Multipole Methods [3]) but at the price of a loss of accuracy/stability. Second, even if boundary element methods provide an accurate solution, the precision is

\footnotetext{
1 xavier.antoine@univ-lorraine.fr
} 
limited since linear finite element spaces are used as well as low-order surface descriptions. Going to higher order basis functions is very complicate and time consuming, most particularly when one wants to integrate with high accuracy (hyper)singular potentials that are involved in an integral formulation.

When the geometry is more trivial, then further simplifications can be realized in the integral equation methods. Indeed, for example, analytical expressions of the integral operators can be obtained, and spectrally accurate and fast solutions can be derived. This is the case when considering a disk. The Matlab toolbox $\mu$-diff considers the case of a collection of $M$ homogeneous circular cylinders where Fourier basis expansions can be used. Even if disks can be considered as simple geometries, they are involved in many physics problems which need a reliable and highly accurate solution most particularly when $k$ and $M$ are large. This is for example the case for photonics and phononics modeling [4] or plasmonic resonance from metallic nanoparticules [5].

\section{FORMULATION AND NUMERICAL SOLUTION USED IN $\mu$-DIFF FOR MULTIPLE SCATTERING PROBLEMS BY DISKS}

To simplify the presentation, let us consider that we wish to solve the sound-soft scattering problem by using a singlelayer representation formula of $(1)$ in $L^{2}(\Gamma)$ written in a variational way: find $\rho \in L^{2}(\Gamma)$ such that

$$
\forall \Phi \in L^{2}(\Gamma),\langle L \rho, \Phi\rangle_{L^{2}(\Gamma)}=\left\langle-u^{i n c}, \Phi\right\rangle_{L^{2}(\Gamma)}
$$

where $L$ is the single-layer operator

$$
\forall \mathbf{x} \in \Gamma, \quad L \rho(\mathbf{x})=\int_{\Gamma} \frac{i}{4} H_{0}^{(1)}(k|\mathbf{x}-\mathbf{y}|) \rho(\mathbf{y}) d \Gamma(\mathbf{y})
$$

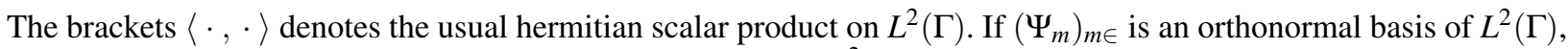
the weak formulation can be written equivalently as: find $\rho \in L^{2}(\Gamma)$ such that

$$
\forall m \in,\left\langle L \rho, \Psi_{m}\right\rangle_{L^{2}(\Gamma)}=\left\langle-\left.u^{i n c}\right|_{\Gamma}, \Psi_{m}\right\rangle_{L^{2}(\Gamma)}
$$

Since we are considering circular boundaries $\Gamma_{p}$, a suitable choice of orthonormal basis is the Fourier basis. More precisely, the spectral approximation that is used in $\mu$-diff is built by using $M$ Fourier basis (one per scatterer) to get a global orthonormal basis of $L^{2}\left(\Gamma_{1}\right) \times \ldots \times L^{2}\left(\Gamma_{M}\right)$. Each scatterer $\Omega_{p}^{-}, p=1, \ldots, M$, is a circular cylinder with center $\mathbf{O}_{p}$ and radius $a_{p}$. A point $\mathbf{x} \in R^{2}$ is given by its local polar coordinates associated with the scatterer $\mathbf{r}_{p}(\mathbf{x})=\mathbf{O}_{p} \mathbf{x}$, $r_{p}(\mathbf{x})=\left|\mathbf{r}_{p}(\mathbf{x})\right|, \theta_{p}(\mathbf{x})=\operatorname{Angle}\left(O x_{1}, \mathbf{r}_{p}(\mathbf{x})\right)$. For each scatterer $\Omega_{p}^{-}, p=1, \ldots, M$, we introduce the Fourier basis functions $\left(\varphi_{m}^{p}\right)_{m \in Z}$

$$
\forall m \in Z, \quad \forall \mathbf{x} \in \Gamma_{p}, \quad \varphi_{m}^{p}(\mathbf{x})=\frac{e^{i m \theta_{p}(\mathbf{x})}}{\sqrt{2 \pi a_{p}}} .
$$

This family provides an orthonormal basis of $L^{2}\left(\Gamma_{p}\right)$. We can gather these $M$ families by introducing $\left(\Phi_{m}^{p}\right)_{m \in Z, p=1, \ldots, M}$ defined by: $\forall p, q \in\{1, \ldots, M\}, \forall m \in Z,\left.\Phi_{m}^{p}\right|_{\Gamma_{q}}=\varphi_{m}^{p}$, if $p=q$, and 0 otherwise. Finally, $\mathscr{B}=\left(\Phi_{m}^{p}\right)_{m \in, p=1, \ldots, M}$ is an orthonormal basis of $L^{2}(\Gamma)$ that we call Fourier basis. To express (3) in $\mathscr{B}$, the density $\rho$ is decomposed as: $\rho=\sum_{p=1}^{M} \sum_{m \in Z} \rho_{m}^{p} \Phi_{m}^{p}$. The weak formulation is then: find the Fourier coefficients $\left(\rho_{n}^{q}\right)_{q=1, \ldots, M, n \in Z}$ such that $\forall p \in\{1, \ldots, M\}, \forall m \in Z, \sum_{q=1}^{M} \sum_{n \in Z} L_{m, n}^{p, q} \rho_{n}^{q}=f_{m}^{p}$. The coefficients $f_{m}^{p}$ of the incident wave can be obtained analytically through a short calculation $[6,7]$. Concerning the coefficients $L_{m, n}^{p, q}$, for the case of the diagonal blocks $(p=q)$, we can prove [6,7] that for $p=1, \ldots, M$, and $m, n \in Z$, we have $L_{m, n}^{p, p}=\delta_{m n} \frac{i \pi a_{p}}{2} J_{m}\left(k a_{p}\right) H_{m}^{(1)}\left(k a_{p}\right)$, where $\delta_{m n}$ denotes the Krönecker symbol and the functions $J_{m}$ and $H_{m}^{(1)}$ are respectively the Bessel and first-kind Hankel functions of order $m$. For the off-diagonal terms, we can prove that: for all $1 \leq p \neq q \leq M$, and for all $m, n$ in $Z$, we have: $L_{m, n}^{p, q}=\frac{i \pi \sqrt{a_{p} a_{q}}}{2} J_{m}\left(k a_{p}\right) J_{n}\left(k a_{q}\right) H_{n-m}^{(1)}\left(k b_{p q}\right) e^{i(n-m) \alpha_{p q}}$, with $b_{p q}=\left|\mathbf{O}_{q} \mathbf{O}_{p}\right|, 1 \leq p \neq q \leq M$ and $\alpha_{p q}=\operatorname{Angle}\left(O x_{1}, \mathbf{b}_{p q}\right)$. We can rewrite the infinite system as: $\widetilde{L} \widetilde{\rho}=\widetilde{\mathbf{f}}$, with the following block structure

$$
\left(\begin{array}{cccc}
\widetilde{L}^{1,1} & \ldots & \widetilde{L}^{1, M} \\
\vdots & \vdots & \ddots & \vdots \\
\widetilde{L}^{M, 1} & \ldots & \widetilde{L}^{M, M} &
\end{array}\right)\left(\begin{array}{c}
\widetilde{\rho}^{1} \\
\vdots \\
\widetilde{\rho}^{M}
\end{array}\right)=\left(\begin{array}{c}
\widetilde{\mathbf{f}}^{1} \\
\vdots \\
\widetilde{\mathbf{f}}^{M}
\end{array}\right),
$$


where $\widetilde{L}^{p, q}=\left(L_{m, n}^{p, q}\right)_{m, n \in Z}$, for $1 \leq p, q \leq M$. A rapid analysis shows that each block can be written as a product of diagonal and Toeplitz matrices involving special functions. The unknown infinite vector $\widetilde{\rho}^{p}=\left(\rho_{m}^{p}\right)_{m \in Z}$, for $p=$ $1, \ldots, M$, contains the coefficients of the unknown $\rho$ in the Fourier basis of $L^{2}\left(\Gamma_{p}\right)$. The right-hand side infinite vector $\widetilde{\mathbf{f}}^{p}=\left(f_{m}^{p}\right)_{m \in}$, for $p=1, \ldots, M$, is the vector of Fourier coefficients of the trace of $-u^{i n c}$ on $\Gamma_{p}$. Other source terms could be considered.

The infinite linear system (4) is next truncated in the Fourier space thanks to an adaptive process that involves the wavenumber $k$ and the radii $a_{p}$ leading to a spectrally accurate solution. The finite-dimensional linear system is accurately solved in $\mu$-diff by using a direct solver or a preconditioned Krylov subspace linear solver that uses fast matrix-vector products based on FFTs, the solution depending on the configuration with respect to $k a_{p}$ and $M$. Low memory is also considered when $k a_{p}$ is large enough since the storage of the Toeplitz matrices can be optimized. Because $\mu$-diff includes all the integral operators that are needed in scattering (traces and normal derivative traces of the single- and double-layer potentials), a large class of scattering problems can be solved. Concerning the geometrical configurations, any deterministic or random distribution of disks is possible. Finally, $\mu$-diff includes post-processing facilities like e.g.: surface and far-fields computations, total and scattered exterior (near-field) visualization...

An example of computation with $\mu$-diff is given on Figure 1. We report the real part of the total acoustic near-field and the far-field pattern when illuminating 100 randomly distributed circular cylinders (with $a_{p} \approx 0.1$ ) by a point source located at $(-5,0)$ with $k=2 \pi$. The obstacles are penetrable with a random index varying between 1.2 and 1.7.

\section{ACKNOWLEDGMENTS}

The second, third and fourth authors would like to thank the Institute of Scientific Research and Revival of Islamic Heritage at Umm Al-Qura University (Project ID 43405027) for the financial support.

\section{REFERENCES}

1. P. Martin, Multiple scattering. Interaction of time-harmonic waves with $N$ obstacles, Cambridge University Press, Cambridge, 2006.

2. Y. Saad, Iterative Methods for Sparse Linear Systems, PWS Publishing Company Boston, 1996.

3. L. Greengard, and V. Rokhlin, J. Comput. Phys. 73, 325-348 (1987).

4. R. M. J.D. Joannopoulos, and J. N. Winn, Photonic Crystals: Molding the Flow of Light, Princeton University Press, 1995.

5. P. Hewageegana, and V. Apalkov, Physical Review B 77 (2008).

6. X. Antoine, C. Chniti, and K. Ramdani, J. Comp. Phy. 227, 1754-1771 (2008).
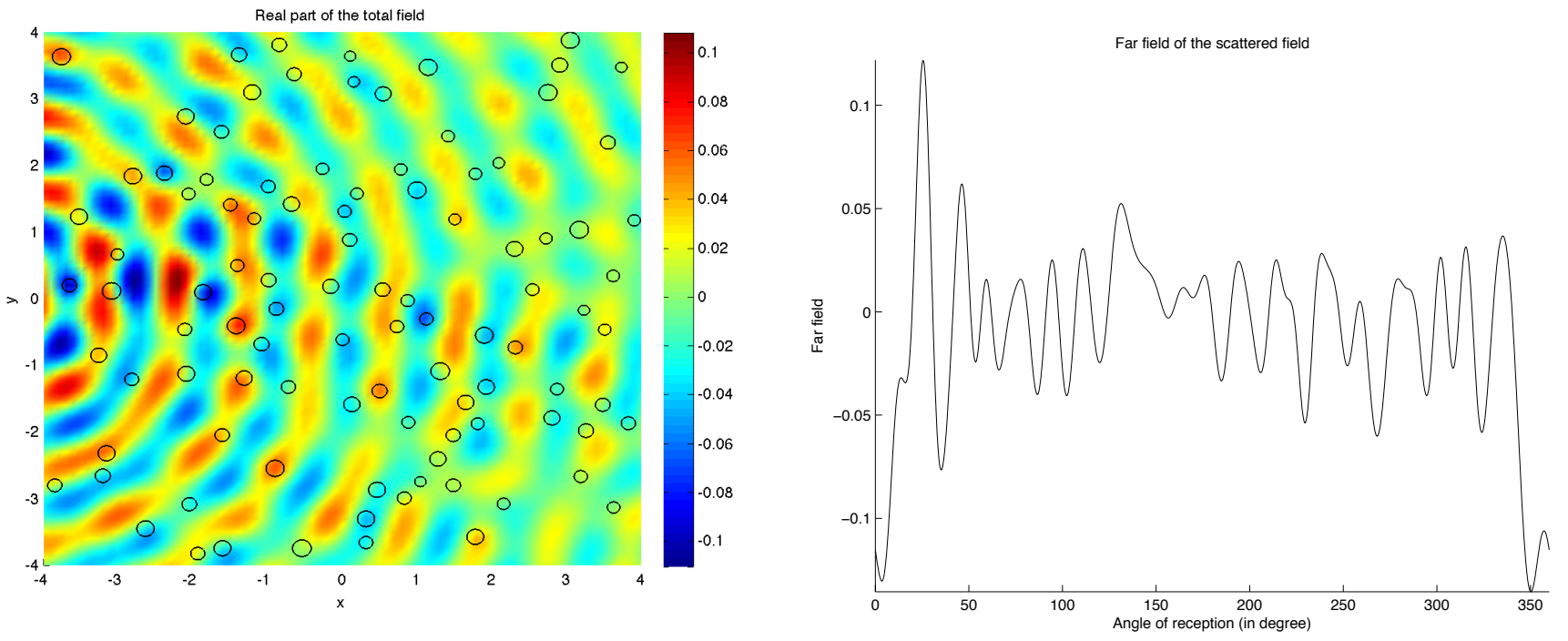

FIGURE 1. Real part of the total acoustic field (left) and far-field pattern (right). 
7. X. Antoine, K. Ramdani, and B. Thierry, J. Algorithms Comput. Technol. 6, 241-259 (2012). 